\title{
Cobalt Telluride: A Highly Efficient Trifunctional Electrocatalyst for Water Splitting and Oxygen Reduction
}

Manashi Nath*a, Umanga De Silva ${ }^{\mathrm{a}}$, Harish Singh ${ }^{\mathrm{a}}$, Matthew Perkins ${ }^{\mathrm{b}}$, Wipula P. R. Liyanage ${ }^{\mathrm{a}}$, Siddesh Umapathi ${ }^{a}$, Shatadru Chakravarty ${ }^{\mathrm{c}}$ Jahangir Masud $^{\mathrm{a}}$,

${ }^{a}$ Department of Chemistry, Missouri University of Science and Technology, Rolla, MO 65409, USA.

${ }^{b}$ Department of Chemical and Biochemical Engineering, Missouri University of Science and Technology, Rolla, MO 65409, USA.

${ }^{c}$ Materials Research Center, Missouri University of Science and Technology, Rolla, MO 65409, USA.

*Email: nathm@mst.edu

Table S1. EDS elemental ratios of electrodeposited and hydrothermally synthesized cobalt tellurides.

\begin{tabular}{|c|c|c|c|c|}
\hline \multirow{2}{*}{ Elements } & \multicolumn{2}{|c|}{ Electrodeposited } & \multicolumn{2}{c|}{ Hydrothermally synthesized } \\
\cline { 2 - 5 } & CoTe & CoTe 2 & CoTe & CoTe $_{2}$ \\
\hline Te L & 54.26 & 67.78 & 53.14 & 65.42 \\
\hline Co K & 45.74 & 32.22 & 46.86 & 34.58 \\
\hline Total & 100.00 & 100.00 & 100.00 & 100.00 \\
\hline
\end{tabular}

\begin{tabular}{|c|c|c|c|c|}
\hline Sample & $\mathrm{K}-139(\mathrm{ppb})$ & Fe-1 $57(\mathrm{ppb})$ & Co 59 (ppb) & Te $125(\mathrm{ppb})$ \\
\hline $\mathrm{KOH}$ (as-prepared) & 39.4 & 1.4 & $<\mathrm{DL}$ & $<\mathrm{DL}$ \\
\hline $\begin{array}{l}\text { Purified KOH after } 12 \mathrm{~h} \\
\text { chronoamperometry with } \\
\text { CoTe }\end{array}$ & 25.2 & $<\mathrm{DL}$ & $<\mathrm{DL}$ & $<\mathrm{DL}$ \\
\hline $\begin{array}{l}\text { Purified KOH after } 12 \mathrm{~h} \\
\text { chronoamperometry with } \\
\mathrm{CoTe}_{2}\end{array}$ & 30.1 & $<\mathrm{DL}$ & $<\mathrm{DL}$ & $<\mathrm{DL}$ \\
\hline
\end{tabular}

$* \mathrm{DL}=$ Detection limit. 

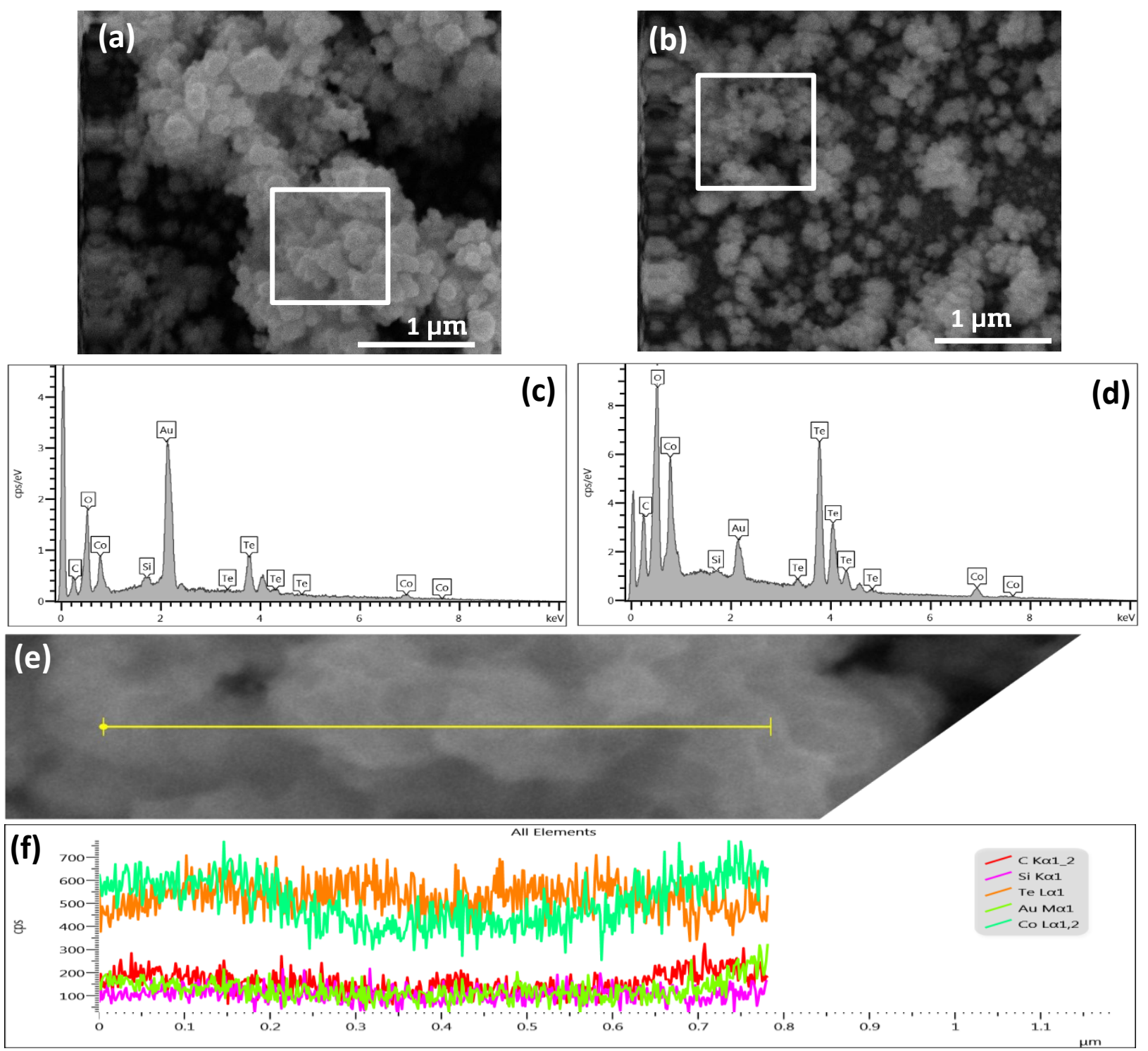

(g)

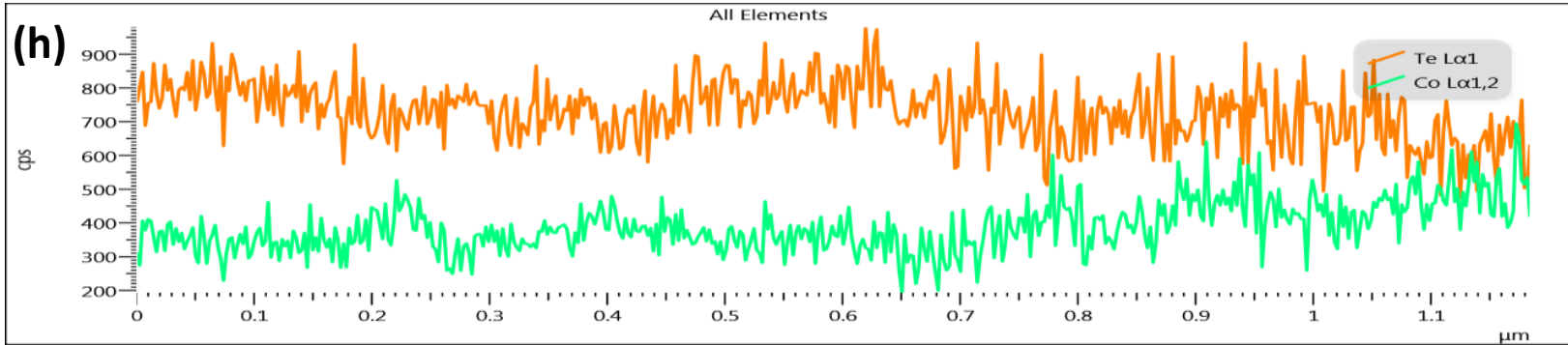

Figure S1. SEM images of electrodeposited (a) CoTe, (b) CoTe 2 . EDS spectra of electrodeposited (c) CoTe, (d) $\mathrm{CoTe}_{2}$. EDS line scan analysis of (e) CoTe, (g) CoTe 2 with corresponding elemental distribution shown in (f) $\mathrm{CoTe}$ and (h) $\mathrm{CoTe}_{2}$. 

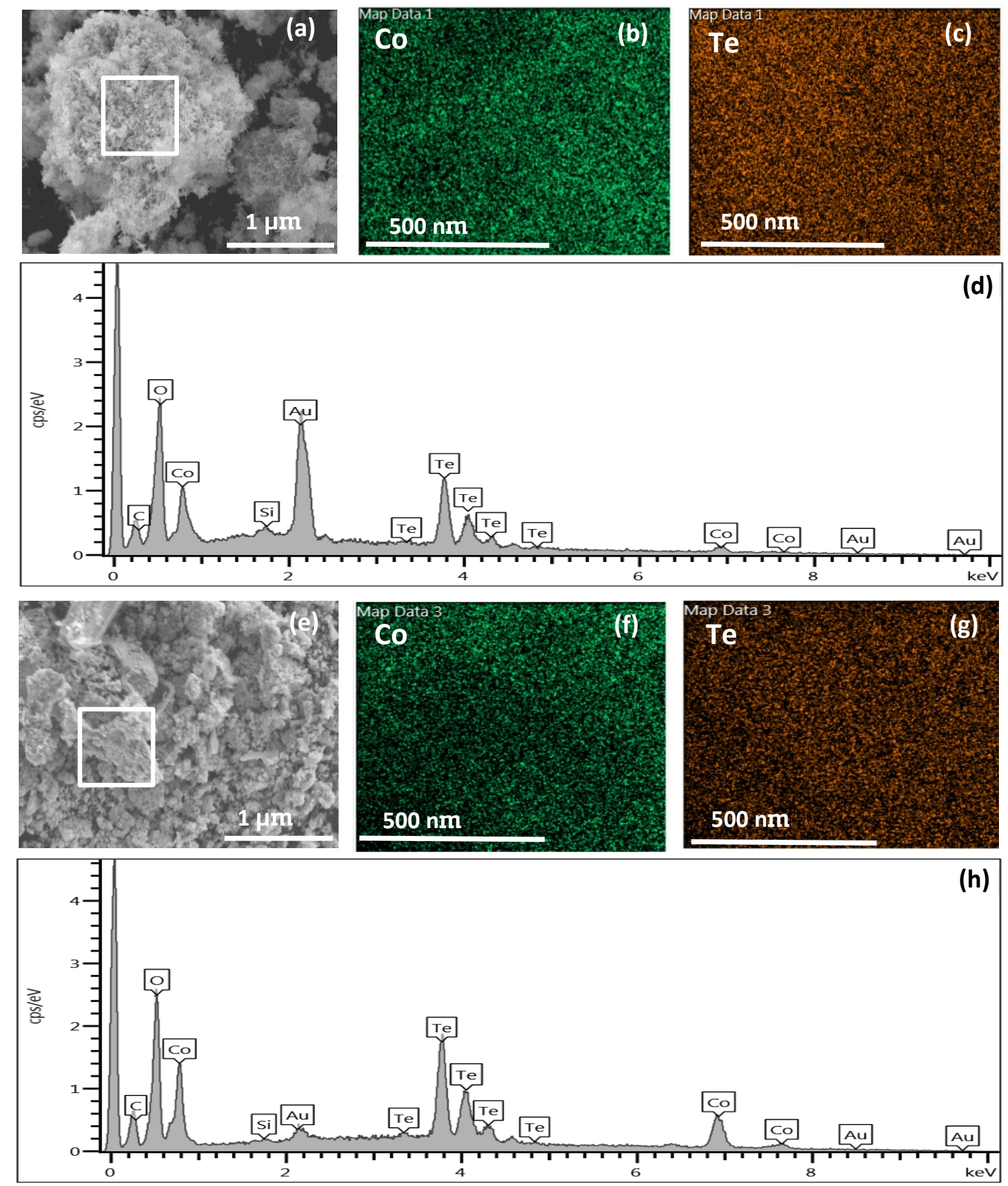

Figure S2. SEM images of hydrothermally synthesized (a) CoTe, (e) CoTe 2 . EDS elemental mapping of (b) Co and (c) Te in CoTe and (f) Co and (g) Te in CoTe 2 respectively. EDS spectra of hydrothermally synthesized (d) CoTe, (h) $\mathrm{CoTe}_{2}$. 

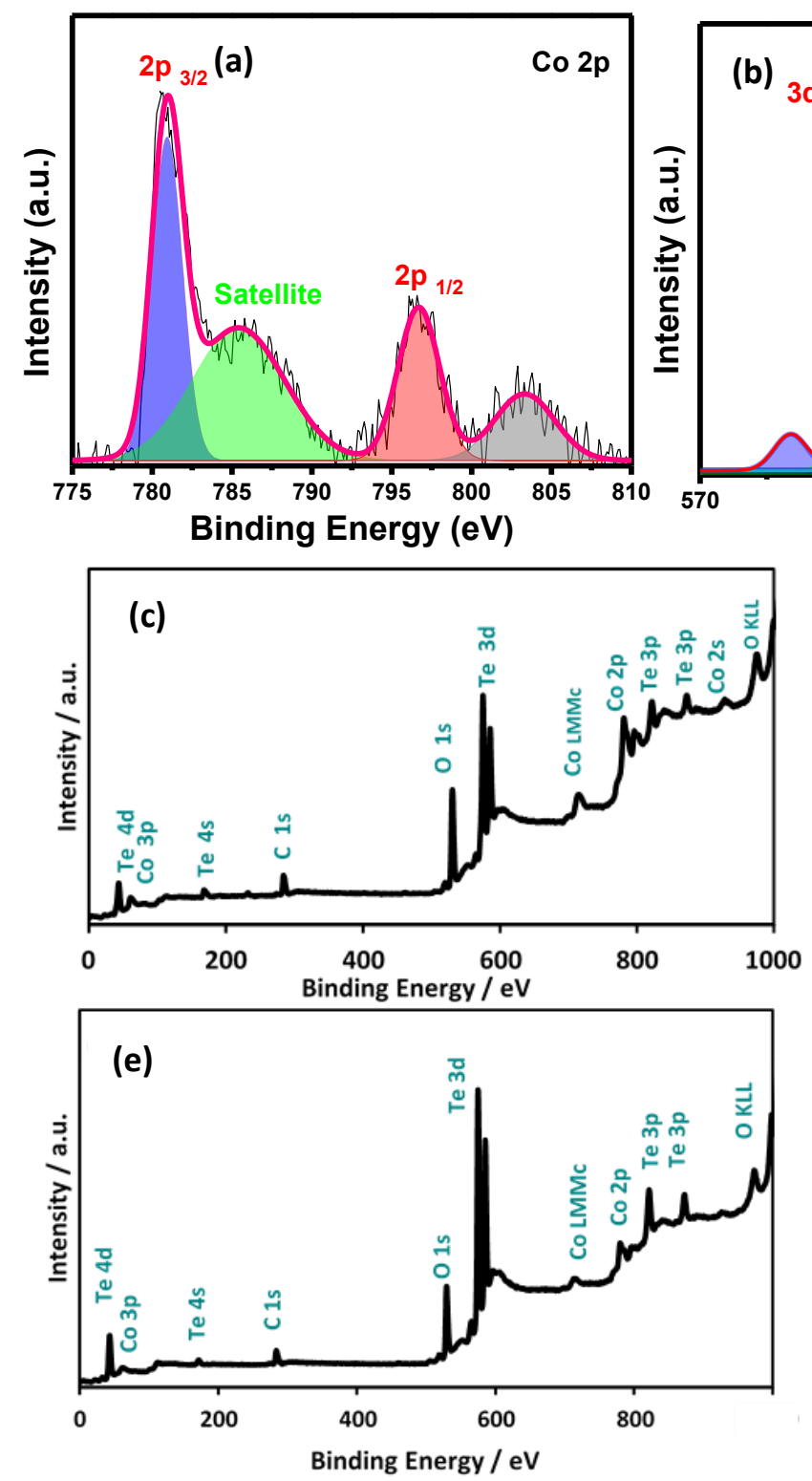
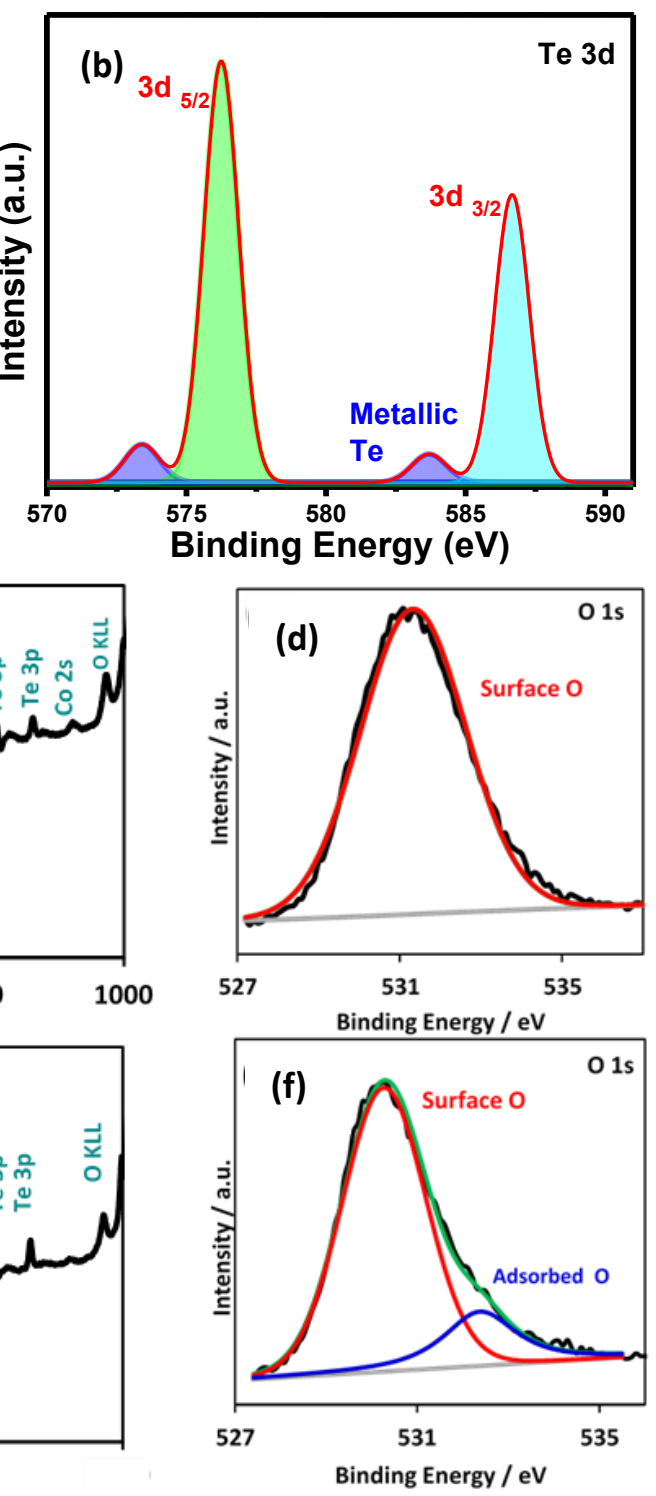

Figure S3. X-ray photoelectron spectroscopy of powdered catalysts of CoTe and CoTe 2 . (a) Co $2 \mathrm{p}$ and (b) Te $3 \mathrm{~d}$ spectra from electrodeposited CoTe. (c) Full-scale survey, and (d) O 1s for CoTe. (e) Full-scale survey, and (f) $\mathrm{O}$ 1s for $\mathrm{CoTe}_{2}$. 


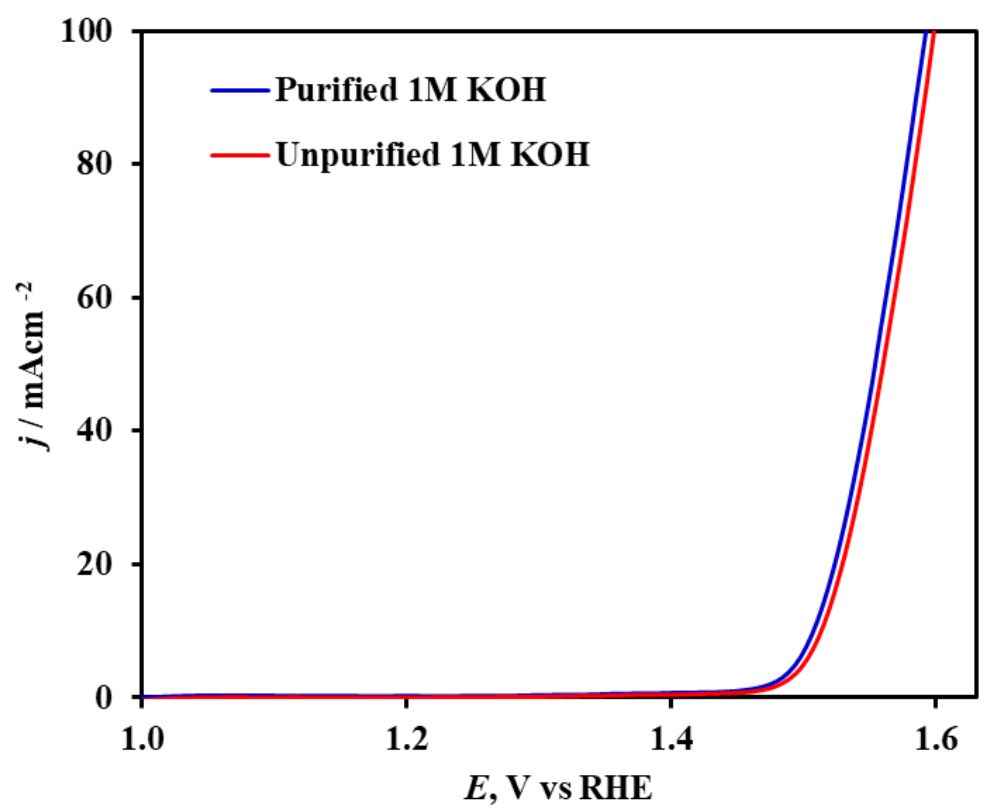

Figure S4. Comparison of LSVs obtained for CoTe-modified electrode in as-prepared $\mathrm{KOH}$ and "purified" $\mathrm{KOH}$ confirming that trace Fe impurity in the electrolyte does not affect OER activity.
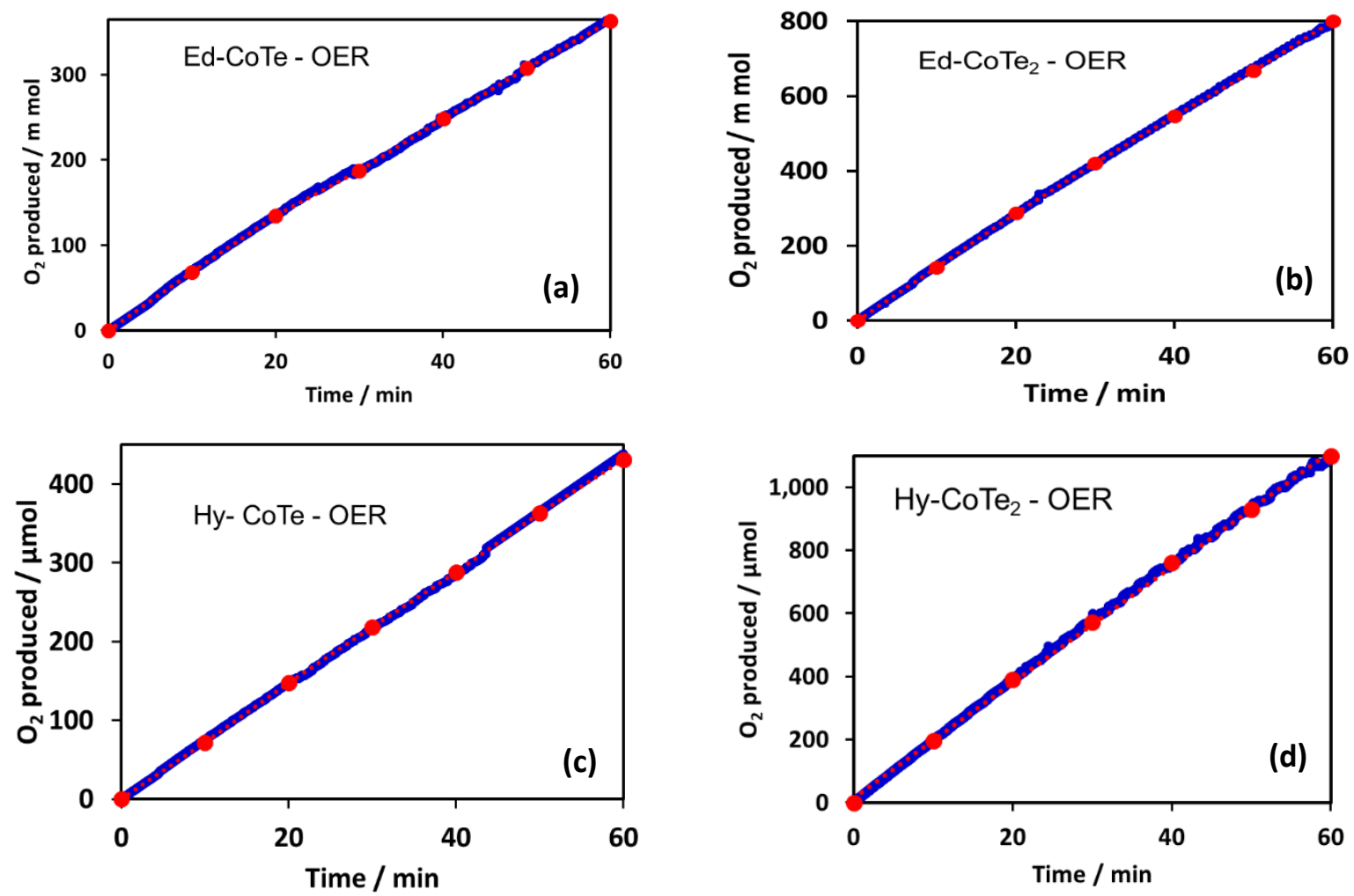

Figure S5. The comparison of experimentally obtained volumes of oxygen with the theoretically calculated amounts for: electrodeposited (a) $\mathrm{CoTe}$, (b) $\mathrm{CoTe}_{2}$; and hydrothermally synthesized (c) $\mathrm{CoTe}$, (d) $\mathrm{CoTe}_{2}$. 


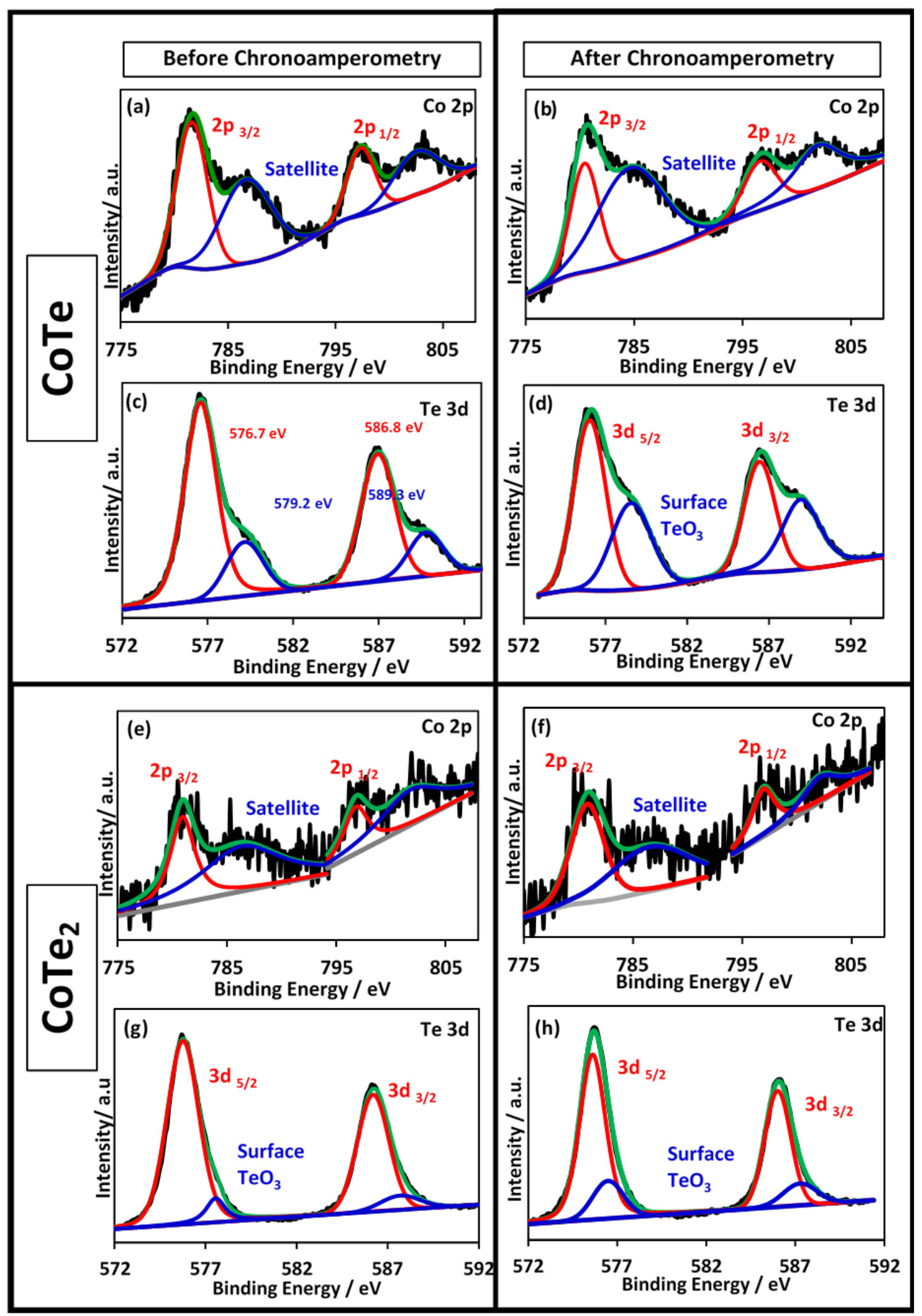

Figure S6. Comparison of XPS spectra before and after chronoamperometry in (a-d) CoTe and (e-h) $\mathrm{CoTe}_{2}$. (a), (e) and (b), (f) Co 2p XPS peak before and after chronoamperometry, respectively; (c), (g) and (d), (h) Te $3 \mathrm{~d}$ peak before and after chronoamperometry, respectively. 

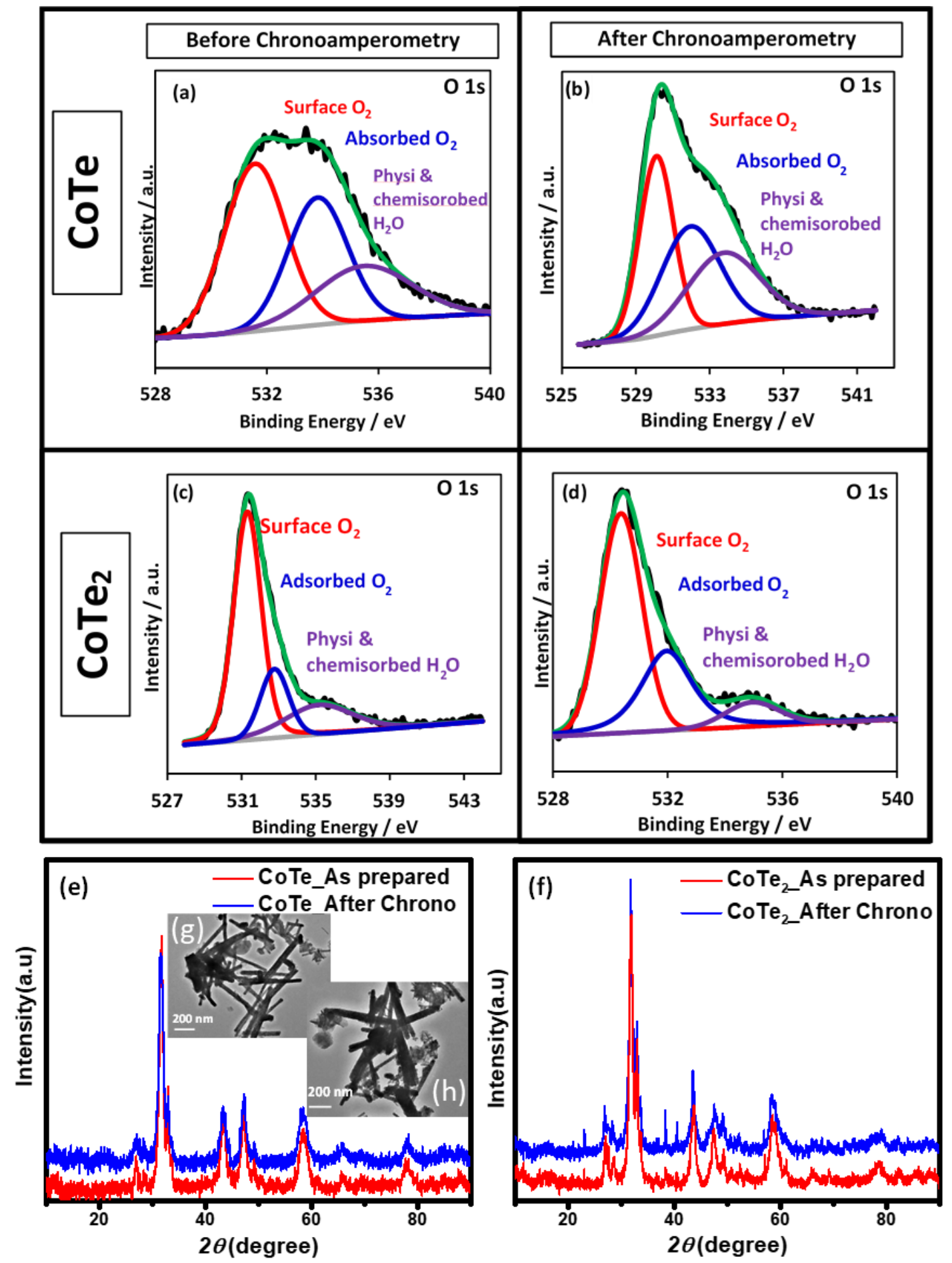

Figure S7. O 1s XPS spectra from CoTe-modified electrodes (a) before chronoamperometry and (b) after chronoamperometry. O 1s XPS spectra from CoTe-modified electrodes (c) before chronoamperometry (d) after chronoamperometry. XRD of hydrothermally synthesized (e) CoTe and (f) $\mathrm{CoTe}_{2}$. Inset of Figure (e) shows TEM of Hydrothermally synthesized CoTe (g) before and (h) after chronoamperometry. 

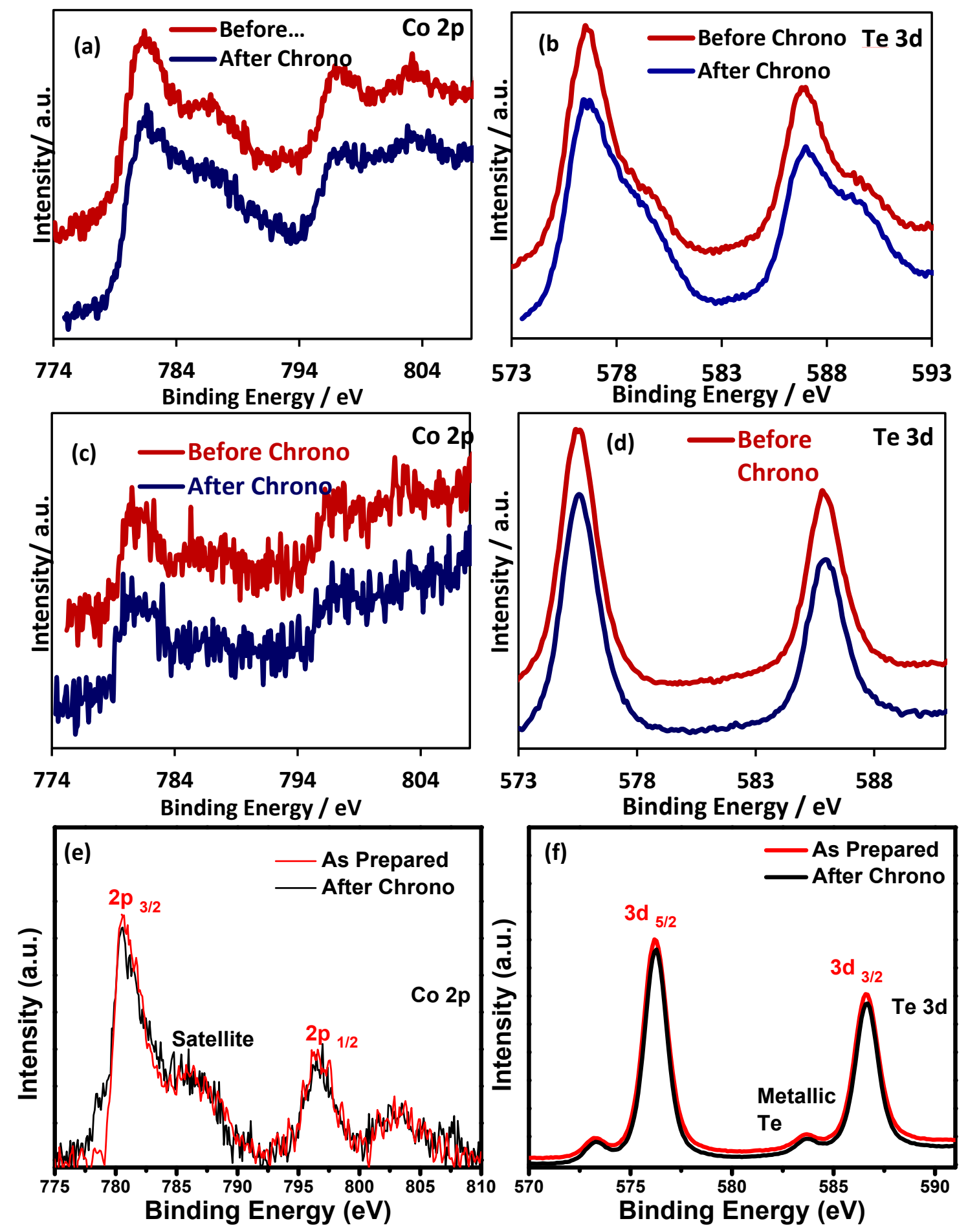

Figure S8. The comparison of XPS spectra before and after chronoamperometry for hydrothermally synthesized CoTe (a) Co 2p, (b) Te 3d; and CoTe 2 (c) Co 2p and (d) Te 3d. Comparison of XPS after chronoamperometry for electrodeposited CoTe (e) Co $2 p$ and (f) Te $3 d$. 

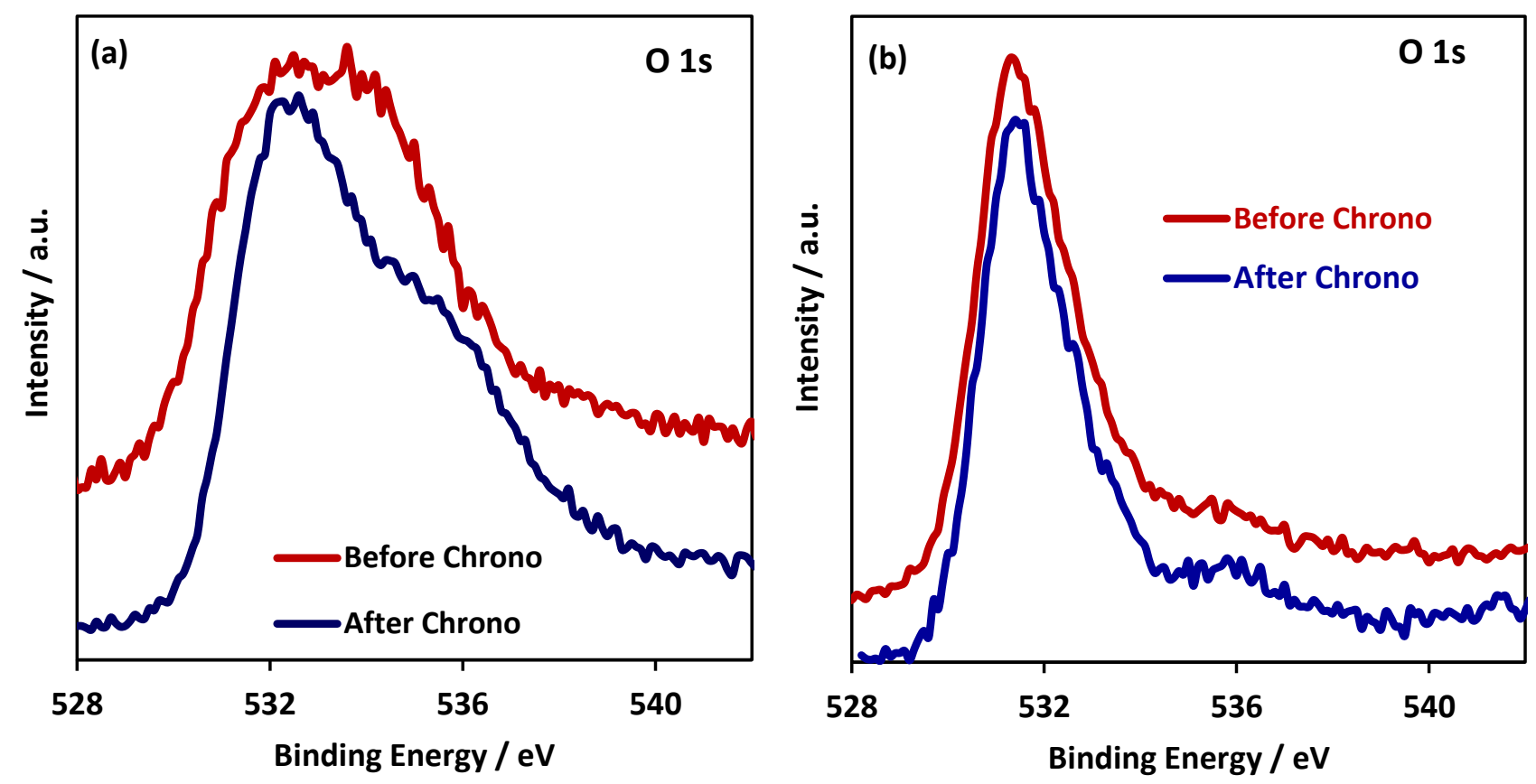

Figure S9. The comparison of O 1s XPS spectra before and after chronoamperometry for (a) CoTe and (b) $\mathrm{CoTe}_{2}$. 

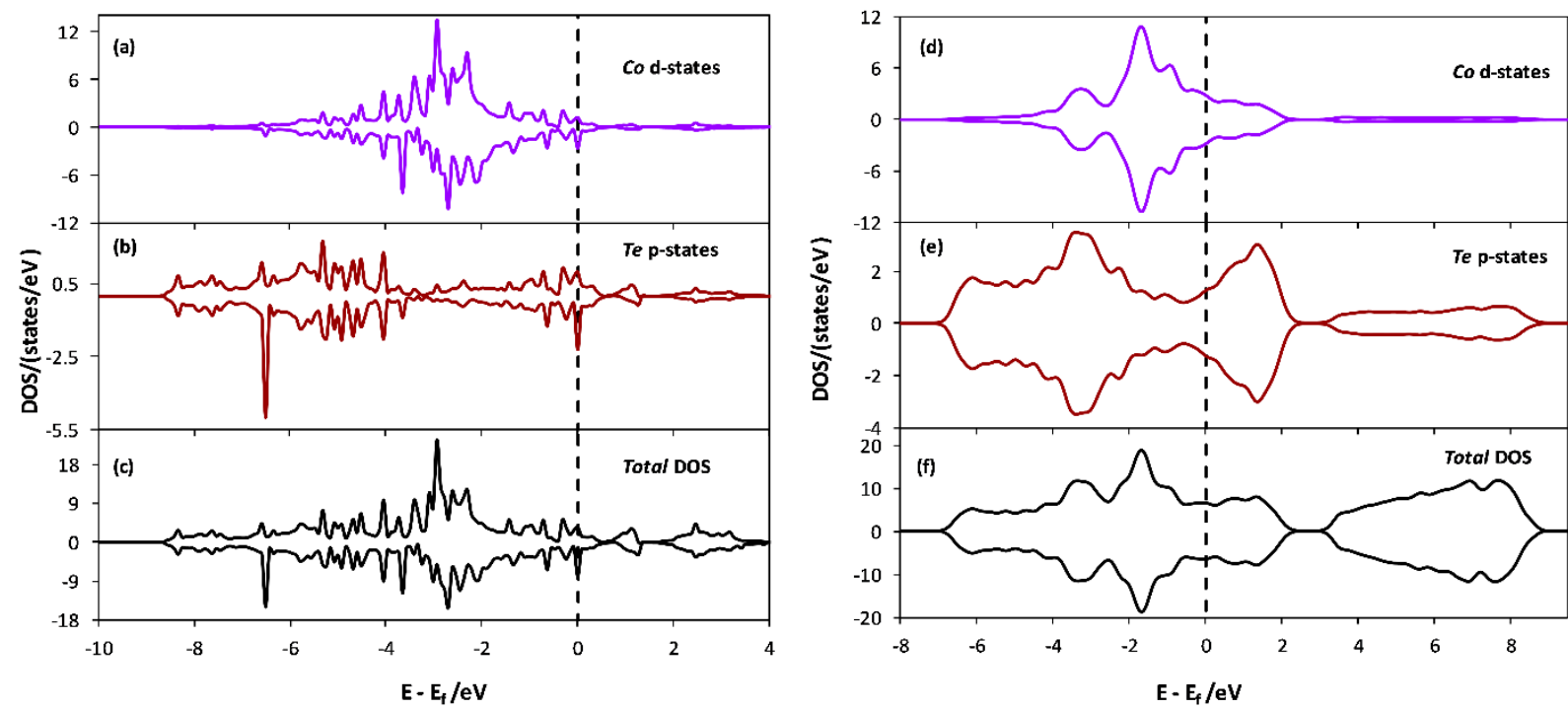

Figure S10. Contribution from (a) Co d-states and (b) Te p-states to the (c) total density of states (DOS) of CoTe in (001) surface. Accordingly, (d) and (e) illustrates the contribution from Co d-states and Te p-states to the (f) DOS of CoTe 2 (001) surface.
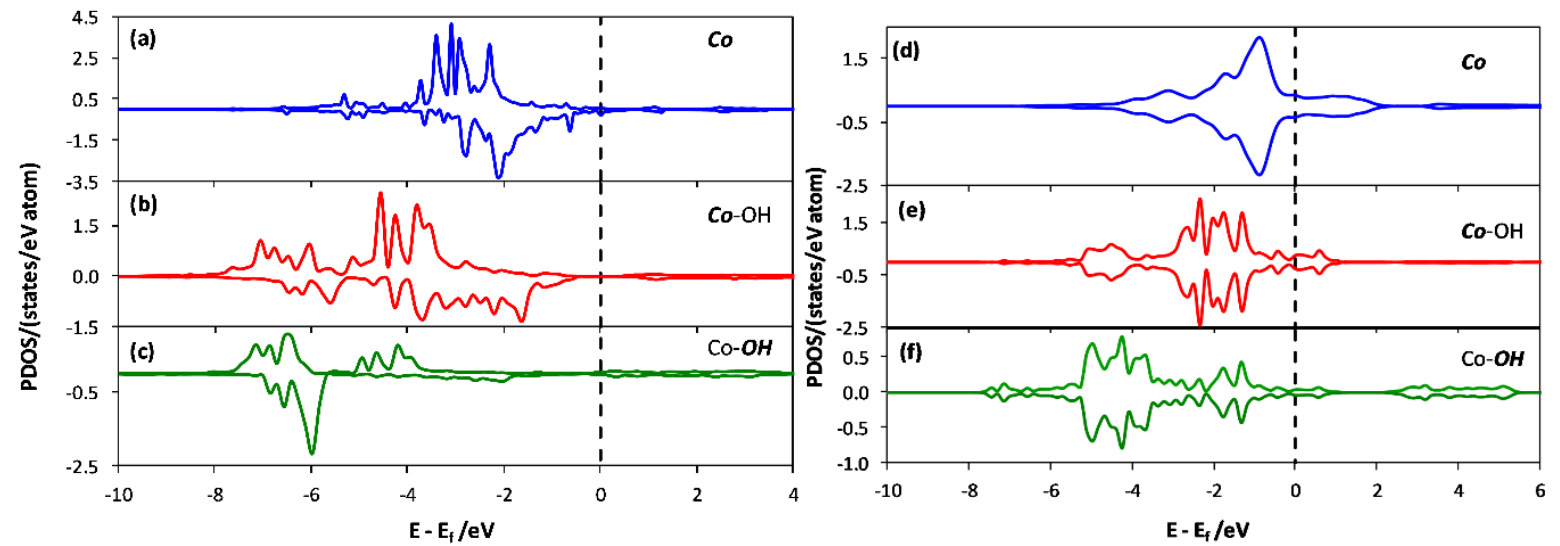

Figure S11. Calculated partial density of states (PDOS) of CoTe in (001) surface (a) Co atom before the attachment of $\mathrm{OH}^{-}$ion, (b) $\mathrm{Co}$ atom after the attachment of $\mathrm{OH}^{-}$ion, (c) $\mathrm{OH}^{-}$ions after attachment on Co atom. Similarly, PDOS of $\mathrm{CoTe}_{2}$ (001) surface (d) Co atom before the attachment of $\mathrm{OH}^{-}$ion, (e) $\mathrm{Co}$ atom after the attachment of $\mathrm{OH}^{-}$ion and (f) $\mathrm{OH}^{-}$ions after attachment on Co atom. 
Table S3: Comparison of electrocatalytic parameters for the HER

\begin{tabular}{|l|c|c|c|c|c|}
\hline & CoTe-ED & CoTe2-ED & CoTe-HD & CoTe2-HD $_{2}$ & Pt \\
\hline Onset Potential / V & -0.195 & -0.175 & -0.207 & -0.250 & 0.000 \\
\hline$\eta$ to $10 \mathrm{~mA} \mathrm{~cm}^{-2} / \mathrm{mV}$ & 283 & 350 & 270 & 378 & 52 \\
\hline Tafel slope $/ \mathrm{mV} \mathrm{dec}^{-1}$ & 72.8 & 90.5 & 57.2 & 96.5 & 38.5 \\
\hline
\end{tabular}
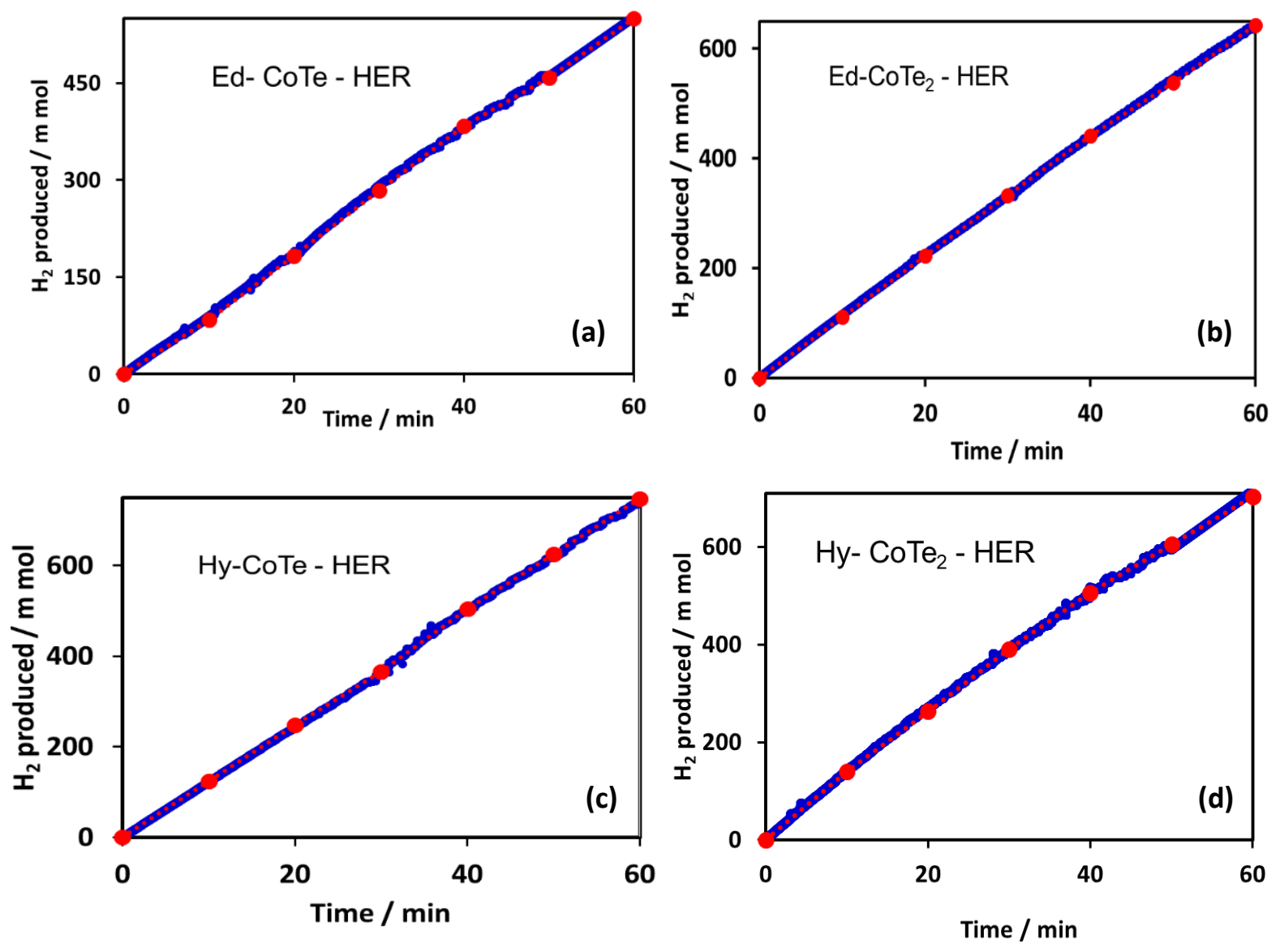

Figure S12. The comparisons of experimentally obtained volumes of hydrogen with the theoretically calculated amounts: electrodeposited (a) $\mathrm{CoTe}$, (b) $\mathrm{CoTe}_{2}$; hydrothermally synthesized (c) CoTe, (d) $\mathrm{CoTe}_{2}$. 

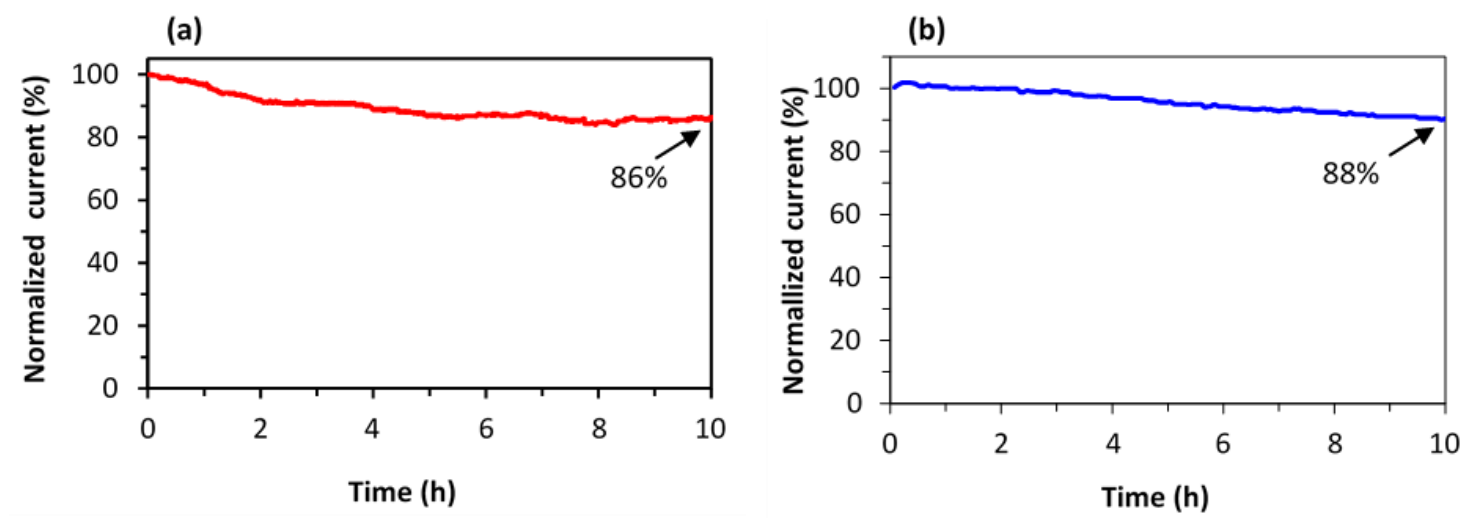

Figure S13. Stability of the (a) CoTe and (b) $\mathrm{CoTe}_{2}$ electrocatalysts $\left(0.6 \mathrm{mg} / \mathrm{cm}^{2}\right.$ loading) under continuous ORR conditions in $\mathrm{O}_{2}$ saturated $0.1 \mathrm{M} \mathrm{NaOH}$ solution at $400 \mathrm{rpm}$ on RRDE.

Table S4: The comparison of energy efficiency of cobalt telluride catalysts.

\begin{tabular}{|c|c|c|c|c|}
\hline Catalysts & $\begin{array}{c}\operatorname{OER}(\mathrm{V})^{\mathrm{a}} \text { at } \\
\text { at } 10 \mathrm{~mA} \mathrm{~cm}^{-2}\end{array}$ & $\begin{array}{c}\operatorname{HER}(\mathrm{V})^{\mathrm{a}} \text { at } \\
\text { at } 10 \mathrm{~mA} \mathrm{~cm}^{-2}\end{array}$ & $\begin{array}{l}\text { Water splitting } \\
\text { cell voltage at } \\
\text { at } 10 \mathrm{~mA} \mathrm{~cm}^{-2}\end{array}$ & $\begin{array}{c}\text { Energy } \\
\text { efficiency }(\%)^{b}\end{array}$ \\
\hline ED- CoTe & 1.43 & 0.28 & 1.71 & 86.54 \\
\hline ED- $\mathrm{CoTe}_{2}$ & 1.47 & 0.35 & 1.82 & 81.31 \\
\hline HD- CoTe & 1.50 & 0.27 & 1.77 & 83.61 \\
\hline HD- $\mathrm{CoTe}_{2}$ & 1.54 & 0.38 & 1.92 & 77.08 \\
\hline
\end{tabular}


Table S5: The comparison of OER activity of reported cobalt telluride catalysts

\begin{tabular}{|c|c|c|c|c|c|c|}
\hline OER Catalysts & Electrolytes & $\begin{array}{c}\text { Onset } \\
\text { potential } \\
\mathrm{V}\end{array}$ & 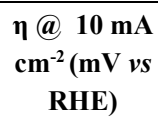 & $\begin{array}{l}\text { Tafel slope } \\
\text { mV dec }\end{array}$ & $\begin{array}{c}\text { Loading } \\
\left(\mathrm{mg} \mathrm{cm}^{-2}\right)\end{array}$ & Reference \\
\hline $1-\mathrm{D}-\mathrm{CoTe}-200{ }^{\circ} \mathrm{C}$ & 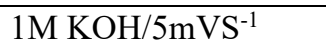 & 1.51 & 370 & - & 0.7 & 1 \\
\hline $\mathrm{CoTe}_{2}$ & \multirow[t]{2}{*}{$0.1 \mathrm{~m} \mathrm{KOH}$} & $1.53^{*}$ & 357 & 32 & 0.25 & \multirow[t]{2}{*}{2} \\
\hline CoTe & & $1.51 *$ & 365 & 73 & 0.25 & \\
\hline $\mathrm{CoTe}_{2} \mathrm{NA} / \mathrm{TM}$ & $1.0 \mathrm{M} \mathrm{KOH}$ & $1.50 *$ & $340^{\mathrm{b}}$ & 67 & 1.45 & 3 \\
\hline CoTe & 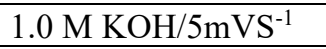 & 1.56 & 370 & - & - & 4 \\
\hline $\mathrm{CoTe}_{2} / \mathrm{CoOx}$ & $1.0 \mathrm{M} \mathrm{KOH} / 10 \mathrm{mVS}^{-1}$ & $1.45^{*}$ & 380 & 58 & 0.28 & 5 \\
\hline $\mathrm{CoTe}_{2} @ \mathrm{NCNTFs}$ & $1.0 \mathrm{M} \mathrm{KOH}$ & 1.56 & 330 & 98.6 & 0.285 & 6 \\
\hline $\mathrm{CoTe}_{2} / \mathrm{CNT}$ & $1.0 \mathrm{M} \mathrm{KOH}$ & $1.50 *$ & 291 & 44.2 & - & 7 \\
\hline $\mathrm{CoTe}$ & $1.0 \mathrm{M} \mathrm{KOH}$ & 1.51 & 420 & - & 0.9 & 1 \\
\hline $\mathrm{CoTe}_{2} @ \mathrm{NCNTFs}$ & $1.0 \mathrm{M} \mathrm{KOH}$ & $1.53 *$ & 330 & 82.8 & 0.285 & 6 \\
\hline ED- CoTe & $1.0 \mathrm{M} \mathrm{KOH} / 10 \mathrm{mVS}^{-1}$ & 1.44 & 200 & 43.8 & \multirow[t]{4}{*}{6.25} & \multirow{4}{*}{$\begin{array}{l}\text { This } \\
\text { work }\end{array}$} \\
\hline HD- CoTe & $1.0 \mathrm{M} \mathrm{KOH} / 10 \mathrm{mVS}^{-1}$ & 1.44 & 270 & 67.7 & & \\
\hline ED- $\mathrm{CoTe}_{2}$ & $1.0 \mathrm{M} \mathrm{KOH} / 10 \mathrm{mVS}^{-1}$ & 1.48 & 240 & 44.8 & & \\
\hline $\mathrm{HD}-\mathrm{CoTe}_{2}$ & 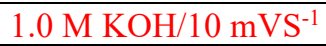 & 1.43 & 320 & 111.8 & & \\
\hline $\mathrm{ED}-\mathrm{RuO}_{2}$ & $1.0 \mathrm{M} \mathrm{KOH} / 10 \mathrm{mVS}^{-1}$ & 1.51 & 370 & - & - & $\begin{array}{l}\text { This } \\
\text { Work }\end{array}$ \\
\hline
\end{tabular}

Table S6: The comparison of HER activity of reported cobalt telluride catalysts

\begin{tabular}{|c|c|c|c|c|c|c|}
\hline HER Catalysts & Electrolytes & $\begin{array}{c}\text { Onset } \\
\text { potential } \\
V\end{array}$ & $\begin{array}{c}\eta @ 10 \mathrm{~mA} \\
\mathrm{~cm}^{-2}(\mathrm{mV} v s \\
\mathrm{RHE})\end{array}$ & $\begin{array}{l}\text { Tafel slope } \\
\text { mV dec }\end{array}$ & $\begin{array}{l}\text { Loading } \\
\left(\mathrm{mg} \mathrm{cm}^{-2}\right)\end{array}$ & Reference \\
\hline $\begin{array}{l}\text { CoTe NSs/CoTe } 2 \\
\text { NTs }\end{array}$ & \multirow[t]{3}{*}{$\begin{array}{l}0.5 \mathrm{M} \mathrm{H}_{2} \mathrm{SO}_{4} \\
/ 5 \mathrm{mVS}^{-1}\end{array}$} & $-0.16^{*}$ & -172 & 57.1 & 4.85 & \multirow[t]{3}{*}{8} \\
\hline CoTe NTs & & $-0.24 *$ & -284 & 58.7 & - & \\
\hline $\begin{array}{l}\text { CoTe2 NDs/CoTe } 2 \\
\text { NTs }\end{array}$ & & $-0.27 *$ & -309 & 63.2 & 4.57 & \\
\hline $\mathrm{CoTe}_{2}$ & $\begin{array}{l}0.5 \mathrm{M} \mathrm{H}_{2} \mathrm{SO}_{4} \\
/ 10 \mathrm{mVS}^{-1}\end{array}$ & -0.2 & -246 & 41 & - & 9 \\
\hline \multirow[t]{2}{*}{$\begin{array}{l}\mathrm{CoTe}_{2} @ \text { NCNTFs- } \\
700{ }^{\circ} \mathrm{C}\end{array}$} & $\begin{array}{l}1 \mathrm{M} \mathrm{KOH} / 5 \mathrm{mV} \\
\mathrm{s}^{-1}\end{array}$ & $-0.15^{*}$ & -208 & 58.04 & 0.285 & \multirow[t]{2}{*}{6} \\
\hline & $\begin{array}{l}0.5 \mathrm{M} \mathrm{H}_{2} \mathrm{SO}_{4} / 5 \\
\mathrm{mV} \mathrm{s}^{-1}\end{array}$ & $-0.15 *$ & -240 & 61.67 & 0.285 & \\
\hline $\mathrm{CoTe}-200^{\circ} \mathrm{C}$ & $\begin{array}{l}1 \mathrm{M} \mathrm{KOH} / 5 \mathrm{mV} \\
\mathrm{s}^{-1}\end{array}$ & $-0.50 *$ & $-550 *$ & - & 0.7 & 1 \\
\hline \multirow[t]{2}{*}{ CoTe2@NCNTFs } & $0.5 \mathrm{M} \mathrm{H} 2 \mathrm{SO} 4$ & $-0.2^{*}$ & -240 & 61.67 & \multirow[t]{2}{*}{0.285} & \multirow[t]{2}{*}{6} \\
\hline & $1.0 \mathrm{M} \mathrm{KOH}$ & $-0.2 *$ & -208 & 58.04 & & \\
\hline CoTe-ED & $\begin{array}{l}1.0 \mathrm{M} \mathrm{KOH} / 10 \\
\mathrm{mVS}^{-1}\end{array}$ & -0.195 & 283 & 72.8 & \multirow[t]{4}{*}{6.25} & \multirow[t]{4}{*}{ This work } \\
\hline CoTe - HD & $\begin{array}{l}1.0 \mathrm{M} \mathrm{KOH} / 10 \\
\mathrm{mVS}^{-1}\end{array}$ & -0.207 & 270 & 57.2 & & \\
\hline $\mathrm{CoTe}_{2}-\mathrm{ED}$ & $\begin{array}{l}1.0 \mathrm{M} \mathrm{KOH} / 10 \\
\mathrm{mVS}^{-1}\end{array}$ & -0.175 & 350 & 90.5 & & \\
\hline $\mathrm{CoTe}_{2}-\mathrm{HD}$ & $\begin{array}{l}1.0 \mathrm{M} \mathrm{KOH} / 10 \\
\mathrm{mVS}^{-1}\end{array}$ & -0.250 & 378 & 96.5 & & \\
\hline
\end{tabular}




\section{References}

(1) Kim, E. K.; Bui, H. T.; Shrestha, N. K.; Shin, C. Y.; Patil, S. A.; Khadtare, S.; Bathula, C.; Noh, Y. Y.; Han, S. H. An Enhanced Electrochemical Energy Conversion Behavior of Thermally Treated Thin Film of 1-Dimensional CoTe Synthesized from Aqueous Solution at Room Temperature. Electrochim. Acta 2018, 260, 365-371.

(2) Gao, Q.; Huang, C. Q.; Ju, Y. M.; Gao, M. R.; Liu, J. W.; An, D.; Cui, C. H.; Zheng, Y. R.; Li, W. X.; Yu, S. H. Phase-Selective Syntheses of Cobalt Telluride Nanofleeces for Efficient Oxygen Evolution Catalysts. Angew. Chemie - Int. Ed. 2017, 56 (27), 7769-7773.

(3) Ji, L.; Wang, Z.; Wang, H.; Shi, X.; Asiri, A. M.; Sun, X. Hierarchical CoTe 2 Nanowire Array: An Effective Oxygen Evolution Catalyst in Alkaline Media. ACS Sustain. Chem. Eng. 2018, 6 (4), 4481-4485.

(4) Patil, S. A.; Kim, E. K.; Shrestha, N. K.; Chang, J.; Lee, J. K.; Han, S. H. Formation of Semimetallic Cobalt Telluride Nanotube Film via Anion Exchange Tellurization Strategy in Aqueous Solution for Electrocatalytic Applications. ACS Appl. Mater. Interfaces 2015, 7 (46), 25914-25922.

(5) McKendry, I. G.; Thenuwara, A. C.; Sun, J.; Peng, H.; Perdew, J. P.; Strongin, D. R.; Zdilla, M. J. Water Oxidation Catalyzed by Cobalt Oxide Supported on the Mattagamite Phase of CoTe2. ACS Catal. 2016, 6 (11), 7393-7397.

(6) Wang, X.; Huang, X.; Gao, W.; Tang, Y.; Jiang, P.; Lan, K.; Yang, R.; Wang, B.; Li, R. Metal-Organic Framework Derived $\mathrm{CoTe}_{2}$ Encapsulated in Nitrogen-Doped Carbon Nanotube Frameworks: A High-Efficiency Bifunctional Electrocatalyst for Overall Water Splitting. J. Mater. Chem. A 2018, 6 (8), 3684-3691.

(7) Lu, T. H.; Chen, C. J.; Lu, Y. R.; Dong, C. L.; Liu, R. S. Synergistic-Effect-Controlled CoTe $2 /$ Carbon Nanotube Hybrid Material for Efficient Water Oxidation. J. Phys. Chem. C 2016, 120 (49), 28093-28099.

(8) Wang, K.; Ye, Z.; Liu, C.; Xi, D.; Zhou, C.; Shi, Z.; Xia, H.; Liu, G.; Qiao, G. Morphology-Controllable Synthesis of Cobalt Telluride Branched Nanostructures on Carbon Fiber Paper as Electrocatalysts for Hydrogen Evolution Reaction. ACS Appl. Mater. Interfaces 2016, 8 (5), 2910-2916.

(9) Lu, T. H.; Chen, C. J.; Basu, M.; Ma, C. G.; Liu, R. S. The CoTe2 Nanostructure: An Efficient and Robust Catalyst for Hydrogen Evolution. Chem. Commun. 2015, 51 (95), 17012-17015. 